\title{
TEXTBOOK IMAGES
}

\section{OF A MOLECULE}

\section{By means of noncontact atomic force microscopy it is possible to atomically resolve individual organic molecules. For this purpose, being able to control the atomic structure of the tip apex is crucial. With a CO-terminated tip, the complete chemical structure of a pentacene molecule could be resolved, showing the five fused hexagonal carbon rings and even the $\mathrm{C}-\mathrm{H}$ bonds [1].}

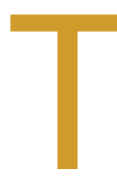

he ability to resolve the atomic structure of single molecules can enable or facilitate studies of various basic chemical and physical processes such as chemical reactions, charge transfer, conformational changes, and molecular adsorption on the level of individual molecules. Application in the fields of surface catalysis, organic photovoltaics, and future molecular electronic devices will benefit from such investigations.

Scanning probe methods are ideally suited for investigations of individual adsorbates, in contrast to diffraction methods which only yield information for ensembles of atoms or molecules. The scanning probe methods that achieve the highest spatial resolution are scanning tunneling microscopy (STM) and its offspring, atomic force microscopy (AFM). Both methods have been successfully employed in the past to image numerous surfaces with atomic resolution, but the atomic structure of an individual molecule has not been resolved until now. To understand the particular difficulties in the imaging of molecules, it is important to review the working principles of STM and AFM.
$\Delta$ Model of a CO functionalized tip above a pentacene molecule. The measured AFM data (frequency shift obtained in constant height mode) is shown as color coded map. 


\section{STM vs. AFM}

In STM, an electrostatic potential $U$ is applied between the tip and the sample and the resulting tunneling current $I_{t}$ is measured. As a consequence, STM is sensitive to electrons with energies lying between the Fermi energy $E_{F}$ and $E_{F}+U$.STM can be used to image molecular frontier orbitals, as has been demonstrated by Repp et al. [2], who recorded fascinating images of the highest occupied molecular orbital (HOMO) and the lowest unoccupied molecular orbital (LUMO) of a

The atomic termination of the tip is crucial for the contrast observed above the molecule. pentacene molecule. This information is of great importance, as it reflects the electronic properties of the molecule. However, the atomic positions that would be revealed by imaging the inner electrons (with energy levels much lower than the Fermi level) are hardly accessible by STM.

Noncontact AFM (NC-AFM) relies on a completely different imaging mechanism. Here the probe is attached to a mechanical resonator, which is oscillated at its resonant frequency. The imaging signal is given by the frequency shift $\Delta f$ of the resonance that arises due to forces acting between the tip and the sample. The energy selection of electrons contributing to STM images does not apply to AFM. However, there are several reasons that render AFM investigations on single

\section{(a)}
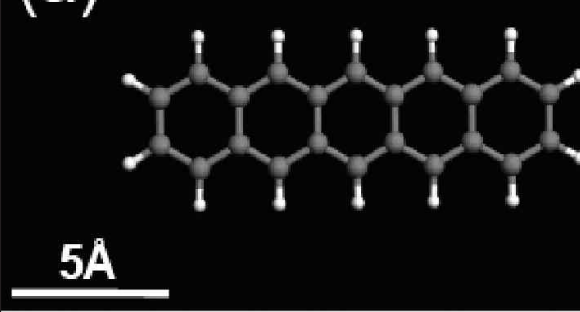

STM and AFM imaging of pentacene on $\mathrm{Cu}(111)$.

(a) Ball-andstick model of the pentacene molecule.

(b) Constantcurrent STM measurement CO-terminated tip, $I_{t}=110 \mathrm{pA}$, $\mathrm{V}=170 \mathrm{mV}$ ).

(c) Constantheight AFM measurement (CO terminated tip, tip height $z$ $=-0.1 \AA$ with respect to the STM set point above $\mathrm{Cu}(111)$, oscillation amplitude $A=0.2 \AA)$. molecules very challenging. One problem is the strong influence of the exact atomic composition and geometry of the tip. In addition, the attraction between the tip and the molecule can lead to unstable imaging conditions, due to displacement or pick-up of the molecule by the tip. Both problems can be solved by functionalizing the tip apex with different atoms and molecules, as will be shown below.

To achieve atomic resolution with AFM, it is necessary to operate in the short-range regime of forces, where chemical interactions give significant contributions. In this force regime, a cantilever of high stiffness is desirable, to enable operation at small oscillation amplitudes (on the order of $1 \AA$ ), as pointed out by Giessibl [3]. In our study, we used a homebuilt combined STM/AFM based on a qPlus sensor design [4] operated in an ultrahigh vacuum at a temperature of $5 \mathrm{~K}$. The high stiffness of the tuning fork [5] sensor allows stable operation at oscillation amplitudes down to $0.2 \AA$. A metal tip was mounted on the free prong of the tuning fork, with a separate tip wire attached to measure the tunneling current.

\section{Pentacene imaging}

The object of our study is pentacene $\left(\mathrm{C}_{22} \mathrm{H}_{14}\right.$, see Fig. 1a), a linear polycyclic hydrocarbon consisting of five fused benzene rings. We imaged pentacene molecules in STM (Fig. 1b) and AFM mode (Fig. 1c) on $\mathrm{Cu}(111)$ after a $\mathrm{CO}$ molecule had been picked up deliberately with the tip. The CO tip termination led to a striking enhancement of the lateral resolution in the AFM mode. The $\mathrm{CO}$ molecule is known to be adsorbed with the carbon atom toward the metal tip and is known to affect STM imaging as well [6]. Several faint maxima and minima are visible in the STM image because of the interaction of the $\mathrm{CO}$ with the pentacene orbitals. In the AFM image (Fig. 1c) the five hexagonal phenyl rings of each pentacene molecule are clearly resolved. We observe local maxima of $\Delta f$ above the edges of the hexagons, near the carbon atom positions, and minima above the centers of the carbon rings (hollow sites). These observations are in concordance with recent AFM measurements on single-walled carbon nanotubes [7]. We also observe the carbon-carbon bonds in the AFM images and even the carbon-hydrogen bonds, indicating the positions of the hydrogen atoms within the pentacene molecule.

In terms of future applications in molecular electronics, insulating substrates will be very important. To prove that this imaging mechanism can indeed be used on insulators, we carried out additional investigations on an insulating $\mathrm{NaCl}$ film of a thickness of two monolayers (ML) on a $\mathrm{Cu}(111)$ substrate (Fig. 2). Moreover, measurements with different atomic tip terminations were performed to study their respective 
effect on the AFM contrast. Using specific protocols to pick up different known adsorbates with the tip apex, we created tips terminated with $\mathrm{Ag}, \mathrm{CO}, \mathrm{Cl}$, and pentacene, and imaged pentacene molecules with these tips as shown in Fig. 2. For each tip, the tip height $z$ was minimized to the closest distance that would still allow stable imaging. Comparing the different tips, we see that the atomic termination is crucial for the contrast observed above the molecule. It should be noted that with metal-terminated tips $(\mathrm{Ag}, \mathrm{Au}$, or $\mathrm{Cu})$, we never observed any atomic contrast, probably because the molecule was always picked up by the tip from the surface before the regime of short-range chemical forces was reached. The highest lateral resolution was observed with $\mathrm{CO}$-modified tips, and in the following we concentrate on this specific tip termination.

\section{Decomposition of forces}

Our AFM measurements yield more than an image of the molecular structure - the actual intermolecular forces can be extracted. AFM can directly quantify forces and energies in processes on the atomic scale. For example, recently the force needed to laterally move a single adatom and the corresponding diffusion barriers have been determined [8], and even the magnetic exchange force was measured with atomic resolution [9]. To extract the force, one needs to integrate $\Delta$ fover $z$, because the measurement value $\Delta f$ is basically proportional to the derivative of the force, with some corrections for the finite value of the oscillation amplitude [10]. Therefore in the experiment, $\Delta f$ has to be recorded as a function of the distance, starting far away (several $\AA$ ) from the surface. We recorded this data in a $3 \mathrm{D}$ field above a pentacene molecule. Figure 3 shows $\Delta f$ and the extracted forces for different tip-sample distances. We observe a featureless molecular image for large distances $(z>2.8 \AA$ ) . With decreasing tip height the contrast increases until we finally observe features on the atomic scale.

Before we try to explain the contrast formation, we want to separate the force contributions between the sample and the CO molecule on the one hand, and between the sample and the rest of the tip on the other. This separation was done experimentally, as shown in Fig. 4. We first measured the force acting on a purely metallic tip (Fig. 4a), then picked up a CO molecule with this tip and measured the force again under otherwise identical conditions (Fig. 4b). The difference of the forces for identical tip positions yielded the estimated contribution from the $\mathrm{CO}$ molecule only (Fig. 4c). Interestingly, we observe that the $\mathrm{CO}$ contribution to the force predominates in the relevant regime, whereas the metallic part of the tip contributes only about $30 \%$ to the attractive forces and yields no corrugation on the atomic scale.

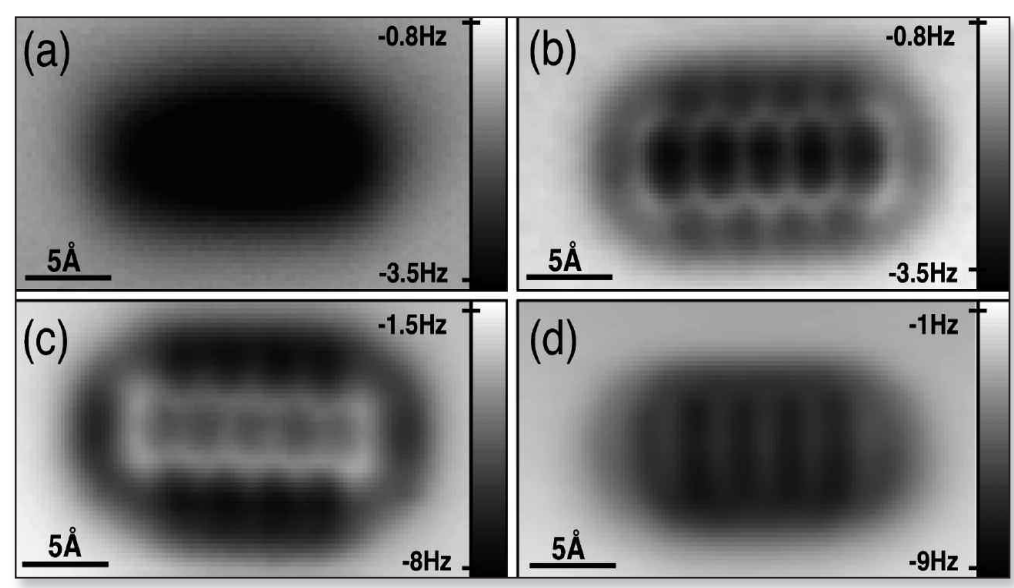

$\Delta$ FIG. 2: Constant-height AFM images of pentacene/ $\mathrm{NaCl}(2 \mathrm{ML}) / \mathrm{Cu}(111)$ using different tip modifications. (a) Ag tip, $z=-0.7 \AA, A=0.6 \AA$; (b) CO tip, $z=+1.3 \AA, A=0.7 \AA$; (c) Cl tip, $z=-1.0 \AA, A=0.7 \AA$, and (d) pentacene tip, $z=+0.6 \AA, A=0.5 \AA$. The $z$-values are given with respect to the STM set point $\left(I_{t}=2 \mathrm{pA}, V=200 \mathrm{mV}\right)$ above the $\mathrm{NaCl}(2 \mathrm{ML}) / \mathrm{Cu}(111)$ substrate.

V FIG. 3: Maps of measured frequency shift $\Delta f(a)$ and extracted vertical force $F_{z}$ (b) at different tip heights $z$. The data shown is part of a complete 3D force field that has been measured in a box of $25 \AA$ by $12.5 \AA$ by $13 \AA ̊$ above a pentacene molecule. The $z$ values are given with respect to the STM set point $\left(I_{t}=2 \mathrm{pA}, V=200 \mathrm{mV}\right)$ above the $\mathrm{NaCl}(2 \mathrm{ML}) / \mathrm{Cu}(111)$ substrate.

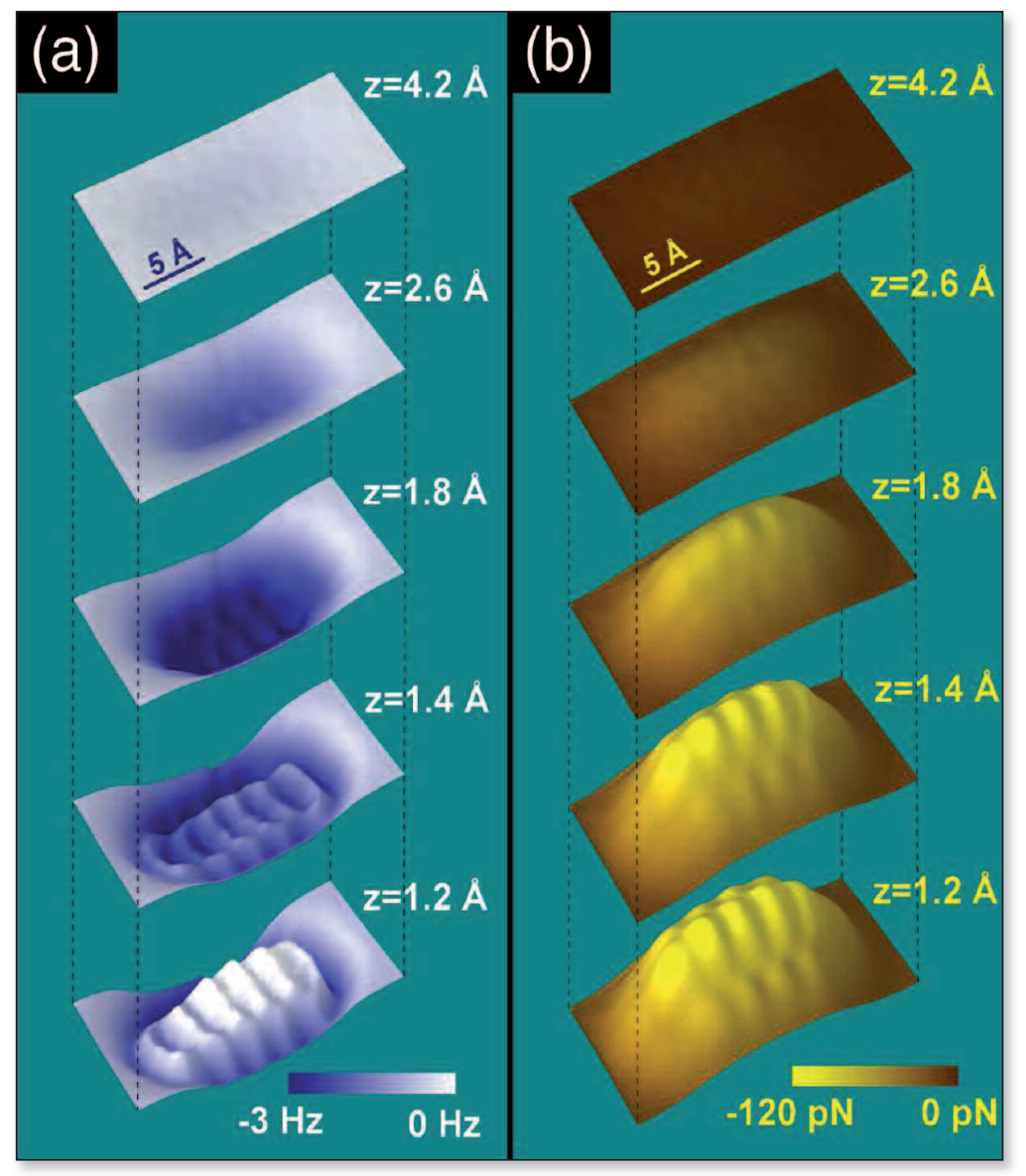

\section{Contrast formation}

A comparison of the extracted forces between the $\mathrm{CO}$ molecule and the pentacene molecule with density functional theory (DFT) calculations yielded a good qualitative and even quantitative agreement [1]. In the calculations we can distinguish forces of three different 
In physical origins: electrostatic forces, van der Waals (vdW) forces, and Pauli repulsive forces. We found that the vdW and electrostatic forces yield a diffuse attractive potential above the entire molecule but show little lateral corrugation on the atomic scale. These contributions give rise to the dark halo surrounding the molecules in the $\Delta \mathrm{f}$ maps. The origin of the atomic contrast is the Pauli repulsion force which becomes significant only when regions of high electron density overlap. These regions are concentrated at the bonds and near the atomic positions and we probe them for sufficiently small tip-sample distances. The AFM image is closely related to the overall electron density, complementary to the STM image, in which the electron orbitals near the Fermi energy are reproduced. Modifying the tip with suitable atomic/molecular terminations was required to operate noncontact AFM in the regime of Pauli repulsion while maintaining stable imaging conditions.
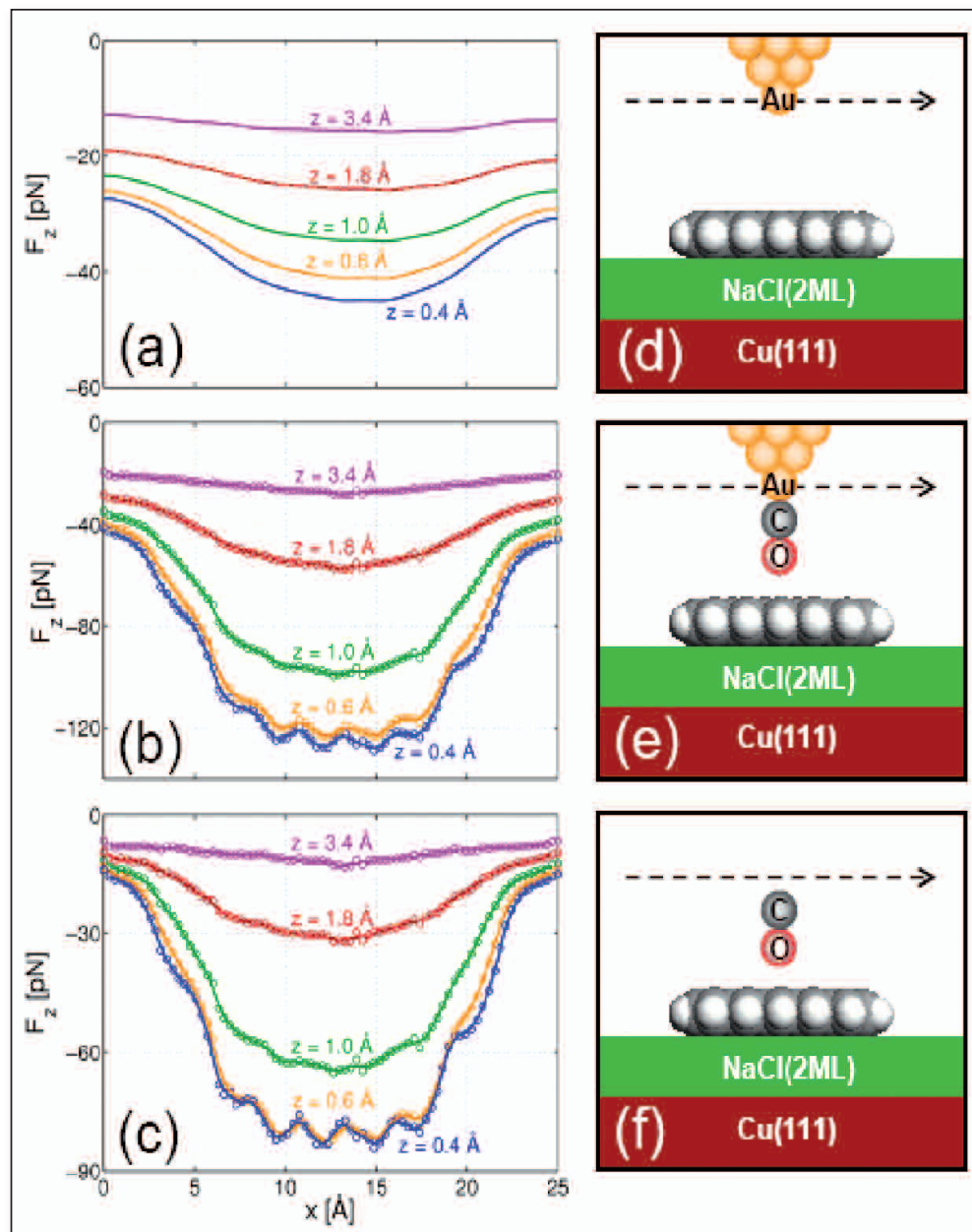

$\Delta$ FIG. 4: Forces above the long axis of a pentacene molecule. (a) Measured with an Au tip and (b) measured with the same tip after picking up a $\mathrm{CO}$ molecule. The $z$-values for both measurements correspond to the same STM-determined set point $\left(I_{t}=2 \mathrm{pA}, V=\right.$ $200 \mathrm{mV}$ for the $\mathrm{CO}$ tip above $\mathrm{NaCl}(2 \mathrm{ML}) / \mathrm{Cu}(111))$. (c) Difference signal: Forces acting on the CO-terminated tip (b) minus the forces of the tip without the $\mathrm{CO}$ molecule (a). The sketches in (d) and (e) depict the measurement geometry of (a) and (b), respectively. (f) Sketch corresponding to the force contribution plotted in (c), i.e. the forces acting on the CO molecule only.

\section{Outlook}

In this work, we successfully employed NC-AFM to image the chemical structure of a molecule revealing the intramolecular bonds and even the hydrogen positions. Going a step further, it may also be possible to extract details about intermolecular bonds, e.g. bond order and length and eventually to probe the reactivity of different molecular sites with respect to the known molecule or atom at the tip apex. Moreover, chemical sensitivity - which was recently demonstrated on semiconductor surfaces [11] - is a goal for future investigations on individual molecules. Furthermore, we aim to combine NC-AFM with electrostatic force microscopy at the atomic level [12] to investigate singleelectron transport and charge distributions in metal-molecule systems.

\section{About the Authors}

Leo Gross works on scanning probe methods and nanofabrication using nanostencil lithography. After he obtained his $\mathrm{PhD}$ at the Freie Universität Berlin he was a post-doc at IBM Research - Zurich, where he is now a research staff member.

Fabian Mohn started his PhD studies in 2008 in the scanning probe group at IBM Research - Zurich, under supervision from the University of Regensburg. Gerhard Meyer leads the low temperature scanning probe group at IBM Research - Zurich. After obtaining his $\mathrm{PhD}$ at the University of Hannover he worked at the IBM Watson Research Center, the Freie Universität Berlin and the Paul-Drude-Institut in Berlin.

\section{References}

[1] L. Gross, F. Mohn, N. Moll, P. Liljeroth, G. Meyer, Science 325, 1110 (2009).

[2] J. Repp, G. Meyer, S. M. Stojkovic, A. Gourdon, C. Joachim, Phys. Rev. Lett. 94, 026803 (2005).

[3] F. J. Giessibl, Rev. Mod. Phys. 75, 957 (2003).

[4] F. J. Giessibl, Appl. Phys. Lett. 76, 1470 (2000).

[5] The parameters of the used tuning fork are: Spring constant $k_{0}$ $\approx 1.8 \times 10^{3} \mathrm{~N} / \mathrm{m}$, resonance frequency $f_{0}=23,165 \mathrm{~Hz}$, quality factor $Q \approx 5 \times 10^{4}$.

[6] L. Bartels, G. Meyer, K.-H. Rieder, D. Velic, E. Knoesel, A. Hotzel, M. Wolf, G. Ertl, Phys. Rev. Lett., 1998, 80, (2004).

[7] M. Ternes, C. P. Lutz, C. F. Hirjibehedin, F. J. Giessibl, A. J. Heinrich, Science 319, 1066 (2008).

[8] M. Ashino, A. Schwarz, T. Behnke, R. Wiesendanger, Phys Rev. Lett. 93, 136101 (2004).

[9] U. Kaiser, A. Schwarz, R. Wiesendanger, Nature 446, 522 (2007).

[10] J. E. Sader, S. P. Jarvis, Appl. Phys. Lett. 84, 1801 (2004).

[11] Y. Sugimoto, P. Pou, M. Abe, P. Jelinek, R. Perez, S. Morita, O. Custance, Nature 446, 64 (2007).

[12] L. Gross, F. Mohn, P. Liljeroth, J. Repp, F. J. Giessibl, G. Meyer, Science 324, 1428 (2009). 